\title{
Animal-assisted therapy for persons with aphasia: A pilot study
}

\author{
Beth L. Macauley, PhD, CCC-SLP, HPCS \\ Department of Communicative Disorders, The University of Alabama, Tuscaloosa, $A L$
}

\begin{abstract}
This study explored the effects and effectiveness of animal-assisted therapy (AAT) for persons with aphasia. Three men with aphasia from left-hemisphere strokes participated in this study. The men received one semester of traditional therapy followed by one semester of AAT. While both therapies were effective, in that each participant met his goals, no significant differences existed between test results following traditional speech-language therapy versus AAT. Results of a client-satisfaction questionnaire, however, indicated that each of the participants was more motivated, enjoyed the therapy sessions more, and felt that the atmosphere of the sessions was lighter and less stressed during AAT compared with traditional therapy.
\end{abstract}

Key words: animal-assisted therapy, aphasia, brain injury, communication, increased motivation, rehabilitation, speechlanguage pathology, speech-language therapy, stroke, therapy animals, therapy efficacy.

\section{INTRODUCTION}

Animals have been used to improve the emotional and functional status of humans since the time of the ancient Greeks [1]. The use of animals ranges from companion animals that provide camaraderie and emotional support to assistance animals that provide direct physicalfunctional support to therapy animals that aid with the habilitation-rehabilitation in physical, occupational, speech-language, and recreation therapy. Therapy animals include turtles, chicks, rabbits, birds, cats, potbellied pigs, fish (aquariums), dogs, and horses. Each animal has specific skills, temperaments, and aptitudes that it brings to the therapy environment. For example, dogs are very friendly and facilitate communication and interaction, while horses offer a riding experience that facilitates the normalization of muscle tone. Within rehabilitation, animals are used more often in counseling, physical therapy, and occupational therapy than in speech-language therapy. Possible reasons for the lack of animal-assisted therapy (AAT) in speech-language therapy include lack of research (1) supporting its use, (2) describing the best ways to incorporate animals in the therapy sessions, (3) determining what types of clients or what types of speech-language disorders respond to the use of animals, and (4) demonstrating the effectiveness of animal therapy compared with traditional speech-language therapy. This study examined the effects and effectiveness of AAT for persons with aphasia.

\section{ANIMAL-ASSISTED THERAPY}

The first documented use of animals in therapy occurred in 1792 at the York Retreat in England, where farm animals were used to improve the attitude of mental

\footnotetext{
Abbreviations: $\mathrm{AAT}=$ animal-assisted therapy, $\mathrm{AD}=$ Alzheimer's disease, $\mathrm{ADL}=$ activities of daily living, $\mathrm{MLU}=$ mean length of utterance, SAFE = Social-Adaptive Functioning Evaluation, WAB = Western Aphasia Battery.

Address all correspondence to Beth $\mathrm{L}$. Macauley, PhD, CCC-SLP, HPCS; Mary K. Chapman Center for Communication Disorders, University of Tulsa, 600 South College, Tulsa, OK 74104; 918-631-2903; fax: 918-631-3668.

Email: beth-macauley@utulsa.edu

DOI: 10.1682/JRRD.2005.01.0027
} 
patients. The founder of nursing, Florence Nightingale, documented the benefits of animals in therapy settings in 1860. She "observed that a small pet is often an excellent companion to the sick" [2]. In the 20th century, animals have been incorporated into numerous healthcare professions, including clinical psychology; nursing; counseling; and recreational, physical, occupational, and speech therapies [3]. AAT has also been conducted in many different environments, including schools, counseling agencies, hospitals, nursing homes, hospice care centers, long-term care centers, residential facilities for persons with severe physical and mental disabilities, juvenile detention centers, and prisons (http://www.deltasociety.org). The incorporation of animals into a mental health program in the United States began in 1919 at St. Elizabeths Hospital in Washington, DC [4].

The integration of AAT into clinical psychology was first credited to the child psychologist, Boris Levinson, who published a paper entitled "The dog as a 'co-therapist"” in Mental Hygiene in 1962 and a book on incorporating animals into child psychology in 1969. Levinson discovered that when his dog Jingles was present during therapy or counseling sessions, significant progress was made when compared with sessions during which Jingles was not present. He went on to find that many children who were withdrawn and uncommunicative would interact positively with the dog [5-6].

Pet visitations are used in the acute-care setting to help establish rapport, facilitate communication, and increase patient responsiveness and social interaction [2]. Through the idea of pet visitation, AAT developed. The Delta Society, an organization formed to promote the benefits of the human-animal bond, formally defines $A A T$ as

\begin{abstract}
A goal-directed intervention in which an animal that meets specific criteria is an integral part of the treatment process. AAT is directed and/or delivered by a health/human service professional with specialized expertise, and within the scope of practice of his/her profession. AAT is designed to promote improvement in human physical, social, emotional, and/or cognitive functioning. AAT is provided in a variety of settings and may be group or individual in nature. This process is documented and evaluated (http://www.deltasociety.org).
\end{abstract}

The Delta Society describes the benefits of AAT to be in the areas of improved empathy, outward focus, nurturing, rapport, acceptance, entertainment, socialization, mental stimulation, physical contact, touch, and physio- logical benefits (http://www.deltasociety.org). More specifically, AAT in hospitals reduced stress among patients and provided both physiological and psychological benefits to the patients [7].

The animals incorporated in animal therapy must be used as a part of the professional's specialty. For example, a speech-language pathologist must use the animal in the context of speech-language therapy, not as a companion pet. The major factor for success with AAT is viewing the animal as a cotherapist rather than an object. This helps develop the relationship between the animal and the patient, creating a bond needed for optimal success [8]. The study of this unique and irreplaceable humananimal bond is at the heart of many different research centers across the United States, such as the Center to Study Human Animal Relationships and Environments at the University of Minnesota, Minneapolis, Minnesota; the Center for Human-Animal Interaction at Virginia Commonwealth University Medical Center, Richmond, Virginia; the Center for Human Animal Relationships at Virginia-Maryland Regional College of Veterinary Medicine, Blacksburg, Virginia; and the Center for the Interaction of Animals and Society within the School of Veterinary Medicine at the University of Pennsylvania, Philadelphia, Pennsylvania.

Although AAT is used in various settings, very little empirical research has documented its effectiveness. Anecdotal reports of the benefits of AAT exist but have not been published in peer-reviewed research journals. Following extensive literature review, we found no study to date that investigated the use of AAT for persons with stroke or aphasia. Despite the lack of research in AAT for persons with aphasia, numerous studies have been performed on the effects of AAT on other adult populations, such as residents of nursing homes [9-12]; patients in acute-care hospitals [13-16]; patients in intensive care units [17]; and adults with spinal cord injury [18], dementia [19-20], depression [21], psychiatric disorders [21-23], and schizophrenia [24-26].

Of the six studies that targeted participants with psychiatric disorders (including schizophrenia), four are worthy of additional discussion. Barker and Dawson examined whether an AAT session reduced the anxiety levels of hospitalized psychiatric patients [22]. In the study, 230 patients participated in a single AAT session and a single traditional recreation therapy session. Before and after participating in the two types of sessions, subjects completed the State-Trait Anxiety Inventory, a self-report measure of anxiety. Barker 
and Dawson used a repeated-measures analysis to test differences in scores from before and after the two types of sessions. Statistically significant reductions in anxiety scores were found after the AAT session for patients with psychotic, mood, and other disorders and after the traditional therapeutic recreation session for patients with mood disorders. The authors concluded that AAT was associated with reduced anxiety levels for hospitalized patients with a variety of psychiatric diagnoses.

Barak et al. evaluated the effects of AAT in a closed psychogeriatric ward over a 12-month period [24]. The participants were 10 elderly persons with schizophrenia and 10 matched control subjects. The AAT consisted of weekly 4-hour sessions that targeted mobility, interpersonal contact, communication, and activities of daily living (ADL) (including personal hygiene and independent selfcare) through the use of cats and dogs as "modeling companions." Raters blind to the participant's group assessed each person with the scale for Social-Adaptive Functioning Evaluation (SAFE) at the beginning and end of the 12 -month period. When the pre- and posttest scores were compared, the AAT group showed more significant improvements than the matched control subjects on both total SAFE score and social functions subscale. The authors concluded that AAT proved a successful tool for enhancing socialization, ADL, and general well-being.

Kovacs et al. studied the effectiveness of AAT in the rehabilitation of schizophrenic patients in a residential social institution [25]. The participants received 9 months of weekly AAT sessions. At the completion of therapy, the participants demonstrated significant improvement in domestic and health activities as assessed by an independent rater using the Independent Living Skills Survey. The authors concluded that AAT was helpful in the rehabilitation of the persons with schizophrenia.

A recent study by Nathans-Barel et al. documented significant improvement in anhedonia for a group of adults with schizophrenia following 10 weeks of AAT when compared with the control group who were treated without AAT [26]. The patients who had the AAT sessions also showed improved use of leisure time and a trend toward improved motivation. The authors concluded that AAT may contribute to the psychosocial rehabilitation and quality of life of persons with schizophrenia.

Of the seven studies that examined AAT for elderly persons with dementia or depression or in nursing homes, four are worthy of additional discussion. Fick studied the effect of AAT on the frequency and types of social interactions among nursing home residents [10]. Point sampling was used to evaluate the behaviors of 36 male nursing home residents at a Department of Veterans Affairs medical center under two conditions: "dog present" and "dog absent." A significant difference in verbal interactions among residents occurred with the dog present. The author concluded that AAT is an effective treatment strategy for increasing socialization among residents in long-term care facilities. Because an increase in social interactions can improve the social climate of an institution, the therapeutic use of animals is a valuable adjunct for reaching treatment goals [11].

Banks and Banks studied the effects of AAT on loneliness for residents of long-term care facilities [11]. They randomized 45 residents into three groups (no AAT, AAT once/week, AAT three times/week; $n=15$ /group). The Demographic and Pet History Questionnaire and version 3 of the University of California at Los Angeles Loneliness Scale were administered before and after a 6-week treatment period. Results indicated that AAT significantly reduced loneliness scores. However, the results of this study must be viewed with caution because each AAT session was conducted by a person and a dog, and the presence of another adult may have contributed to the decrease in loneliness.

Edwards and Beck took a more unique approach and studied whether the presence of fish aquariums influenced the nutritional intake of individuals with Alzheimer's disease (AD) [20]. They studied 62 individuals with $\mathrm{AD}$ who lived in specialized units. Baseline nutritional data were obtained followed by a 2-week treatment period during which the aquariums were introduced. The treatment data were collected daily for 2 weeks and then weekly for 6 weeks. The results indicated that the nutritional intake of the persons with $\mathrm{AD}$ increased significantly when the aquariums were introduced and continued to increase during the 6-week follow-up. Weight also increased significantly over the course of the study. The authors concluded that the presence of the fish aquariums improved the environment of the facilities, thereby improving residents' moods and increasing their desire to eat. The authors also reported that the participants required less nutritional supplementation following the introduction of the fish aquariums, which resulted in healthcare cost savings.

Richeson studied the effects of AAT on the agitated behaviors and social interactions of older adults with 
dementia [12]. Fifteen nursing home residents with dementia participated in daily AAT for 3 weeks, and results indicated a statistically significant decrease in agitated behaviors and increase in social interaction pre- to posttest.

In a unique study of the effects of AAT, Odendaal measured in both people $(n=18)$ and dogs $(n=18)$ six neurochemicals associated with decreased blood pressure before and after positive interaction between the two [27]. Statistically significant results $(p<0.05)$ indicated that the neurochemicals involved in decreasing blood pressure and increasing attention-seeking behavior increased in both species following the interaction. This study provides neurochemical evidence for the reduction of blood pressure and increase in outward focus following AAT.

According to Chapey, persons with aphasia may experience anxiety, depression, frustration, panic, and other mood disorders [28]. Speech-language pathologists, while attempting to improve language function, may actually exacerbate these mood disorders by reminding the persons with aphasia of their speech difficulty. The sudden and severe inability to communicate may render the person unwilling to participate in therapy and cause withdrawal from family and friends. However, the application of AAT to this population targets outward focus, desire to communicate, improvement of mood, decrease in loneliness, and more enjoyable treatment. In addition, many researchers have found that animals exhibit unconditional acceptance through tail wags, facial expressions, purring, and barking, regardless of the person's language difficulties [13]. This helps the patient build confidence. Unfortunately, little research has evaluated the effectiveness of AAT in the clinical setting with a speech-language pathologist.

A literature search, including an annotated bibliography of hippotherapy (the use of equine movement as a treatment strategy) research [29], revealed that six studies to date have examined the effectiveness of AAT within speech-language pathology. All the studies targeted children; four studies examined hippotherapy [30-32] ${ }^{*}$ and two studies examined AAT with a dog [33]. ${ }^{\dagger}$ Therefore, the purpose of this research project was to first examine

\footnotetext{
*Macauley BL. The effects of hippotherapy on respiration and motor speech in persons with cerebral palsy [unpublished master's thesis]. Gainesville (FL): University of Florida; 1989.

${ }^{\dagger}$ Macauley BL, Tanner AK, Laing SP. The effectiveness of animalassisted therapy for preschoolers with language delay: A pilot study. Unpublished observations; 2002.
}

the effects of speech-language AAT for persons with aphasia and second to compare the effectiveness of speech-language AAT with traditional speech-language therapy for persons with aphasia. The following research questions were asked:

1. Is speech-language AAT effective for persons with aphasia?

2. If speech-language AAT is effective, is it less, more, or equally effective as traditional therapy?

3. Will persons with aphasia report differences in their motivation and attitude during traditional versus AAT?

\section{METHODS}

\section{Participants}

The participants for the current study were three men with aphasia from left-hemisphere stroke who were enrolled in speech-language therapy at the Speech and Hearing Center at The University of Alabama, Tuscaloosa, Alabama. Criteria for inclusion included presence of nonfluent aphasia with Western Aphasia Battery (WAB) [34] auditory comprehension scores above 50/60, no allergies to dogs, an affinity to or liking for dogs, and evidence of frustration during speech tasks (e.g., facial expression, refusal to speak, tone of voice). Participant 1 was a 63-year-old man with mild nonfluent aphasia who was 4 years poststroke. His speech was slow and effortful and contained short phrases consisting of content words with good intonation and a mean length of utterance (MLU) of 3.7. His treatment goals included expanding phrases, increasing MLU, improving articulation, and word finding. Participant 2 was a 59-year-old man with moderate nonfluent aphasia who was 7 years poststroke. His speech was also slow and effortful and contained short phrases consisting of nouns with poor intonation and an MLU of 3.1. His treatment goals included verb naming, expanding phrases, and increasing MLU. Participant 3 was a 67-year-old man with severe nonfluent aphasia who was also 7 years poststroke. His speech was very slow and effortful and contained single word responses with an MLU of 1.1. His treatment goals included producing noun-verb phrases, increasing the content of speech by increasing MLU, and decreasing frustration and effort. All participants were living at home with a spouse or caregiver throughout the study. 


\section{Design}

This study implemented a baseline-treatment design with pre-, mid-, and posttesting. To measure the effectiveness of AAT and traditional therapy, we administered both formal and informal measures. The formal measurement consisted of the WAB [34], and the informal measure consisted of a client-satisfaction questionnaire. The questionnaire was designed to measure each participant's motivation and attitude toward the therapy sessions and was derived from a client-satisfaction questionnaire used in a previous study [31]. The questionnaire can be found in Appendix 1 (available online only at http://www.rehab.research.va.gov/). The WAB was administered at the beginning of the first semester of therapy (Time 1), between the two semesters (Time 2), and at the conclusion of the second semester of therapy (Time 3). The questionnaire was administered at Times 2 and 3 only. This study was approved by The University of Alabama Institutional Review Board (03-JCH-005), and signed consent forms were obtained from each participant.

\section{Therapy}

All participants attended one semester of traditional therapy and one semester of AAT. All sessions were individual in nature, 30 minutes in length, and held weekly for 12 weeks. Both the traditional and AAT interventions contained similar activities and targeted similar goals. During the AAT sessions, the participating animal was an 8-year-old neutered male Newfoundland that was a certified Pet Partner ${ }^{\circledR}$ from the Delta Society. A Pet Partner ${ }^{\circledR}$ is an animal that has completed the certification procedures and passed the required behavioral and temperament evaluations from a certified Delta Society Evaluator. Qualifications for a Pet Partner ${ }^{\circledR}$ animal are similar to the Canine Good Citizen criteria from the American Kennel Club and include the obedience commands sit, stay, come, lie down, heel, speak on command, and not jumping on people. Pet Partners ${ }^{\circledR}$ additionally requires that the animals pass temperament standards such as being tolerant of wheelchairs, canes, loud noises (yelling, whistles, and bells), and people who walk with clumsy movements or speak loudly and unintelligibly. The animal must not be skittish, nervous, or afraid when challenged by these environments. The animal must also receive regular grooming and veterinary checks, be current with vaccinations, and be on a flea/tick prevention program.
Both the traditional and AAT sessions were conducted by a graduate student clinician in the Department of Communicative Disorders at the University of Alabama under the supervision of a certified speech-language pathologist who was present in the room. The AAT sessions added a Newfoundland that was certified as a Pet Partner ${ }^{\circledR}$ along with the supervising speech-language pathologist. All therapy and assessment sessions were conducted at the Speech and Hearing Center at The University of Alabama. Examples of therapy activities across traditional and AAT sessions can be found in Appendix 2 (available online only at http://www.rehab.research.va.gov/)

\section{RESULTS}

\section{Research Question 1}

To determine whether speech-language AAT is effective for persons with aphasia, we compared results of the WAB from Times 2 and 3 and evaluated questionnaire responses from Time 3 . While the WAB scores did not change significantly, the questionnaire indicated that all participants believed that they progressed more during the AAT sessions (Table 1). In addition, all participants met or exceeded their therapy goals during the AAT semester, indicating that AAT was effective.

The scores from the questionnaire revealed that each participant reported improvement in speech-language abilities after AAT. When analyzing the questionnaire, we considered responses in the range of 1 to 3 as negative (i.e., regression of skills), responses in the range of 4 to 7 as no improvement (i.e., maintenance of previously learned skills), and responses in the range of 8 to 10 as positive improvement (i.e., active learning and retention of new skills). If a participant circled two numbers, the average of the numbers was used in the calculations (i.e., if a participant circled 8 and 9, then an 8.5 was used). As can be seen in Table 2, the average scores fell in the 5 to 9 range for Time 2, with an overall mean of 7.5, and in the 7 to 10

Table 1.

Western Aphasia Battery Aphasia Quotient scores for each participant before therapy (Time 1), after traditional therapy and before animalassisted therapy (AAT) (Time 2), and after AAT (Time 3).

\begin{tabular}{cccc}
\hline Time & Participant 1 & Participant 2 & Participant 3 \\
\hline 1 & 84.4 & 75.5 & 63.2 \\
2 & 81.1 & 75.1 & 64.8 \\
3 & 83.7 & 76.2 & 66.3 \\
\hline \hline
\end{tabular}


range for Time 3, with an overall mean of 8.3, indicating that the participants believed that AAT helped them improve their ability to communicate.

\section{Research Question 2}

To determine whether AAT is less effective, more effective, or equally effective as traditional clinic-based therapy, we compared the WAB scores from Time 1 to Time 2 and from Time 2 to Time 3. Results indicated little difference on the WAB for each subject across the three measurements (Table 1). However, all participants met or exceeded their therapy goals during both semesters.

With the questionnaire, the differences in each question across Time 2 (posttraditional therapy) and Time 3 (post-AAT) were compared. A difference of less than 2 points was considered negligible, a difference between
2 and 4 points was considered important, and a difference greater than 4 points was considered noteworthy. Please note that the same clinicians conducted both the traditional and AAT sessions. As seen in Table 2, the differences in the participants' responses from Time 2 to Time 3 ranged from 0 to 4.7, with an overall mean difference of 1.3. Within the questionnaire, the responses from questions concerning the clinician (questions 1, 3, 5, 7, 9, $10,11,13,15,17$, and 19) should have remained relatively equal, while the responses for questions concerning the therapy (questions 2, 4, 6, 8, 12, 14, 16, 18, 20, and 21) should have differed. We found this to be true because the mean difference from Time 2 to Time 3 for the clinician questions was 0.5 , while the mean difference from Time 2 to Time 3 for the therapy questions was 2.2. This difference was considered important and

Table 2.

Mean participant responses on client-satisfaction questionnaire after traditional therapy and before animal-assisted therapy (AAT) (Time 2), after AAT (Time 3), and the difference between them. Scale of 1 to 10: 1 = strongly disagree, 10 = strongly agree.

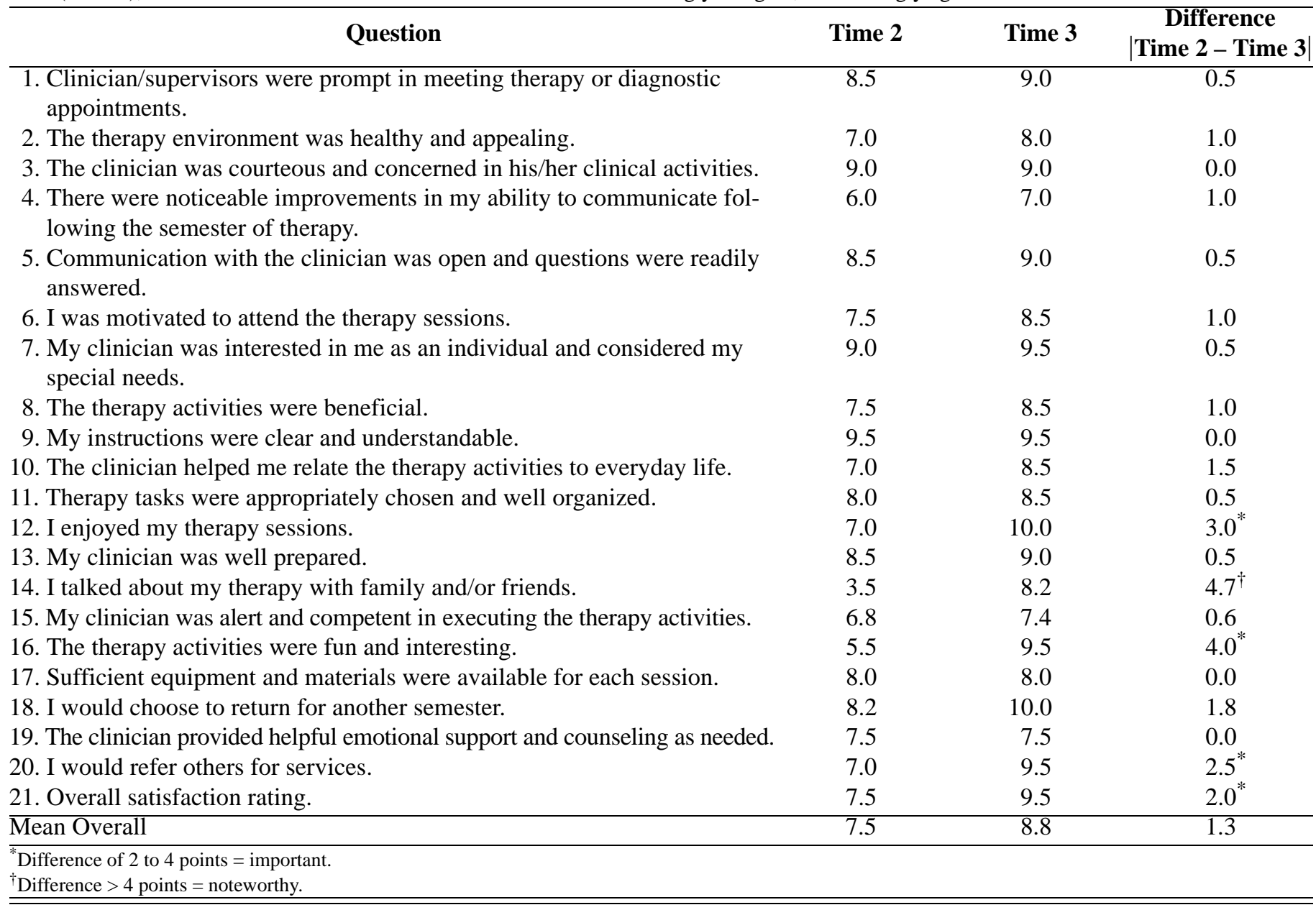


indicated that according to the participants, AAT was more enjoyable than traditional therapy.

\section{Research Question 3}

To determine whether the participants were more motivated to attend therapy during AAT than during traditional therapy, we examined the mean from questions 6, 12, 14, and 16 from Times 2 and 3. As can be seen in Table 2, for these four questions, the participants' mean score was 5.88 , with a range of 3.5 to 7.5 for traditional therapy, and the participants' mean score was 9.05, with a range of 8.5 to 10 for AAT. All participants reported that they were more motivated to attend the therapy sessions when they knew the dog would be present.

\section{DISCUSSION}

Previous research in AAT has examined its efficacy in adult populations, but no studies have examined the benefits of AAT for persons with stroke and aphasia. Results of these previous studies suggest that using animals as an integral part of therapy sessions was effective and motivating. Increased motivation has also been shown in AAT studies with children with language disorders. ${ }^{*}$ The animal used in the majority of these studies was a dog. The purpose of the present study was to investigate the effectiveness of using AAT with adults with aphasia and to determine if AAT was more effective, equally effective, or less effective than traditional speech-language therapy. Results indicated that AAT is effective as a treatment strategy for persons with aphasia and is at least as effective as traditional therapy. Although no differences were noted in formal testing across all conditions, all the participants improved and met their treatment goals each semester. The participants also reported that they enjoyed the AAT sessions more than the traditional sessions and looked forward to their therapy sessions when the dog would be present with greater anticipation. The participants also demonstrated more emotion during the AAT sessions as they initiated communication about their own pets or loss of pets.

\footnotetext{
*Macauley BL, Tanner AK, Laing SP. The effectiveness of animalassisted therapy for preschoolers with language delay: A pilot study. Unpublished observations; 2002.
}

These are important findings for speech-language pathologists who are interested in new and innovative techniques to use with persons with chronic aphasia. This study provides the first empirical evidence that incorporating animals into treatment sessions for speech-language intervention will not sacrifice progress and, in fact, may even facilitate progress toward selected treatment goals. AAT offers the speech-language pathologist an exciting adjunct to traditional therapy by bringing additional creativity and variety to the therapy setting. With animals incorporated into speech-language therapy, the client will be more motivated to attend therapy, more willing to pay attention, and more likely to participate in the therapy activities.

In addition, the dog provides a unique way to decrease the effortfulness that is a hallmark of nonfluent aphasia. That is, the participants spoke with more effort when responding to the clinician than when asked to direct their responses toward the dog, even though in most cases, they were saying the same word or phrase, such as "I am hungry," "Go to Cypress Inn to eat," and "It is raining outside." Clinicians who were not affiliated with the study were asked to observe a session and subjectively decide if the participant spoke with more or less effort when speaking to the clinician than when speaking to the dog. All the observers believed that the participant spoke with more effort toward the clinician and less effort toward the dog. In addition, one observer reported that she believed the participant had improved prosody when speaking to the dog as compared with speaking to the clinician. These findings are consistent with prior studies that reported decreased anxiety and stress when subjects were speaking to an animal versus a person. The change in orientation of turning and speaking to the dog appeared to change the environment of the therapy session. That is, the tension in the air seemed to decrease perceptibly and lightness increased. This environmental change was reported by the participant, the participant's spouse or caregiver who observed the session, and the clinician.

An unexpected benefit of AAT included a trend toward an increased number of spontaneous communicative initiations produced during the sessions. That is, the participants initiated speech more often during the AAT sessions than during the traditional sessions. Most of these initiations were speech directed toward the dog. It makes sense that if a task is perceived as easier and less stressful (e.g., more enjoyable), then a person will initiate 
that task more often. The current investigation included only three persons with chronic nonfluent aphasia. Perhaps a larger study that included more participants, especially those within the first 6 months after brain injury, would reveal statistically significant differences. We should also mention that separating the specific effect of the dog from the nonspecific effects on outcome variables is difficult. That is, the dog was brought into the sessions during the second semester of therapy when the client was already familiar with the clinician. Interaction effects may have also existed between the two treatments (traditional and AAT) since AAT followed traditional therapy for all three participants.

Not surprisingly, the participants in the current study were more likely to direct their communicative initiations toward the dog than the speech-language pathologist. This is an important finding because it documents that the presence of the dog motivates the people to communicate and may even help provide them with something to talk about. Why must our clients with aphasia be motivated to communicate? When clients are hesitant or embarrassed about their speech difficulties, improving their speech is difficult. Talking is a voluntary activity. A clinician cannot use therapeutic techniques designed to expand, extend, and/or model appropriate form, function, or use of language if the client will not provide the language stimulus. The results from the current investigation suggest that the dog may act as a unique catalyst to motivate the client to talk and provide an atmosphere of unconditional acceptance for the disordered speech that is produced.

\section{CONCLUSIONS}

Avenues for future research include determining the effectiveness of AAT for persons with different types of communicative disorders and different types of brain injury. One example of this is to incorporate appropriate and certified animals into speech-language therapy sessions for people with traumatic brain injury or fluent aphasia following stroke. Is AAT equally effective for group treatment as for individual treatment? Does the type of animal incorporated into the therapy session matter? Some clients may be fearful of dogs but respond warmly to cats. Does the presence of a fish aquarium in the treatment room improve the outcome of therapy?
The future research arena for AAT is exciting in its numerous possibilities. We hope that this study will encourage other speech-language pathologists to incorporate Pet Partner ${ }^{\circledR}$ animals into therapy and stimulate further research studies on the effectiveness of AAT in speech-language therapy.

\section{ACKNOWLEDGMENTS}

Dr. Macauley is now with the Mary K. Chapman Center for Communication Disorders, University of Tulsa, Tulsa, Oklahoma.

This material was unfunded at the time of manuscript publication.

The author has declared that no competing interests exist.

\section{REFERENCES}

1. Riede D. The relationship between man and horse with reference to medicine throughout the ages. People, Anim, Environ. 1987;5(2):26-28.

2. Jorgenson J. Therapeutic use of companion animals in health care. Image J Nurs Sch. 1997;29(3):249-54. [PMID: 9378480]

3. Gammonley J, Howie AR, Kirwin S, Zapf SA, Frye J, Freeman G, Stuart-Russell R. Animal-assisted therapy: Therapeutic interventions. Bellevue (WA): Delta Society; 1997.

4. Burch MR. Volunteering with your pet: How to get involved in animal-assisted therapy. New York (NY): Howell Books; 1996.

5. Levinson BM. The dog as a "co-therapist.” Ment Hyg. 1962;46:59-65. [PMID: 14464675]

6. Levinson BM. Pet-oriented child psychotherapy. Springfield (IL): Thomas Publisher; 1969.

7. Carmack BJ, Fila D. Animal-assisted therapy: A nursing intervention. Nurs Manage. 1989;20(5):96,98,100-101. [PMID: 2726087]

8. Kaufmann M. Creature comforts: Animal-assisted activities in education and therapy. Reaching Today's Youth. 1997;1(2):27-31.

9. Gammonley J, Yates J. Pet projects: Animal assisted therapy in nursing homes. J Gerontol Nurs. 1991;17(1):12-15. [PMID: 1899683]

10. Fick K. The influence of an animal on social interactions of nursing home residents in a group setting. Am J Occup Ther. 1993:47(6):529-34. [PMID: 8506934]

11. Banks MR, Banks WA. The effects of animal-assisted therapy on loneliness in an elderly population in long-term care 
facilities. J Gerontol A Biol Sci Med Sci. 2002;57(7): M428-32. [PMID: 12084804]

12. Richeson N. Effects of animal-assisted therapy on agitated behaviors and social interactions of older adults with dementia. Am J Alzheimers Dis Other Demen. 2003;18(6):353-58. [PMID: 14682084]

13. Barba BE. The positive influence of animals: Animalassisted therapy in acute care. Clin Nurse Spec. 1995;9(4): 199-202. [PMID: 7634227]

14. Cole KM, Gawlinski A. Animal-assisted therapy: The human-animal bond. AACN Clin Issues. 2000;11(1):139-49. [PMID: 11040560]

15. Connor K, Miller J. Help from our animal friends. Nurs Manage. 2000;31(7):42-46. [PMID: 15127507]

16. Miller J, Ingram L. Perioperative nursing and animalassisted therapy. AORN J. 2000;72(3):477-83. [PMID: 11004963]

17. Cole KM, Gawlinski A. Animal-assisted therapy in the intensive care unit. A staff nurse's dream comes true. Nurs Clin North Am. 1995;30(3):529-37. [PMID: 7567577]

18. Counsell CM, Abram J, Gilbert M. Animal assisted therapy and the individual with spinal cord injury. SCI Nurs. 1997;14(2):52-55. [PMID: 9295752]

19. Kanamori M, Suzuki M, Yamamoto K, Kanda M, Matsui Y, Kojima E, Fukawa H, Sugita T, Oshiro H. A day care program and evaluation of animal-assisted therapy (AAT) for the elderly with senile dementia. Am J Alzheimers Dis Other Dement. 2000;16(4):234-39. [PMID: 11501346]

20. Edwards NE, Beck AM. Animal-assisted therapy and nutrition in Alzheimer's disease. West J Nurs Res. 2000;24(6): 697-712. [PMID: 12365769]

21. Gress K. Animals helping people. People helping animals. Interview by Shirley A. Smoyak. J Psychosoc Nurs Ment Health Serv. 2003;41(8):18-25. [PMID: 13677008]

22. Barker SB, Dawson KS. The effects of animal-assisted therapy on anxiety ratings of hospitalized psychiatric patients. Psychiatr Serv. 1998;49(6):797-801. [PMID: 9634160]

23. Barker SB, Pandurangi AK, Best A. Effects of animalassisted therapy on patients' anxiety, fear, and depression before ECT. J ECT. 2000;19(1):38-44. [PMID: 12621276]
24. Barak Y, Savorai O, Mavashev S, Beni A. Animal-assisted therapy for elderly schizophrenic patients: A one-year controlled trial. Am J Geriatr Psychiatry. 2001;9(4):439-42. [PMID: 11739071$]$

25. Kovacs Z, Kis R, Rozsa S, Rozsa L. Animal-assisted therapy for middle-aged schizophrenic patients living in a social institution. A pilot study. Clin Rehabil. 2004;18(5): 483-86. [PMID: 15293482]

26. Nathans-Barel I, Feldman P, Berger B, Modai I, Silver H. Animal-assisted therapy ameliorates anhedonia in schizophrenia patients. A controlled pilot study. Psychother Psychosom. 2005;74(1):31-35. [PMID: 15627854$]$

27. Odendaal JS. Animal-assisted therapy-Magic or medicine? J Psychosom Res. 2000;49(4):275-80.

[PMID: 11119784$]$

28. Chapey R. Language intervention strategies in aphasia and related neurogenic communication disorders. 4th ed. Baltimore (MD): Lippincott, Williams \& Wilkins; 2001.

29. Macauley BL. Hippotherapy: An annotated bibliography for research and education. Woodside (CA): American Hippotherapy Association; 2004.

30. Dismuke RP. Rehabilitative horseback riding for children with language disorders. In: Anderson R, Hart BL, Hart LA, editors. The pet connection. Minneapolis (MN): Hawthorne Press; 1984. p. 154-80.

31. Macauley BL, Gutierrez K. The effectiveness of hippotherapy for children with language-learning disabilities. Commun Disord Q. 2004;25(4):205-17.

32. Macauley BL, Drewry C. Hippotherapy facilitates use of an augmentative communication device: A case study. Sci Educ J Ther Riding. 2004;1-8.

33. Adams DL. Animal-assisted enhancement of speech therapy: A case study. Anthrozoos. 1997;10(1):53-56.

34. Kertesz A. Western Aphasia Battery. NewYork (NY): Psychological Corp; 1982.

Submitted for publication January 26, 2005. Accepted in revised form November 3, 2005. 